\title{
AVALIAÇÃO DA QUALIDADE DE SEMENTES DE EUGENIA PLEURANTHA (MYRTACEAE) PELO TESTE DE RAIOS X'.
}

\author{
TATHIANA ELISA MASETTO ${ }^{2}$, ANTONIO CLAUDIO DAVIDE ${ }^{3}$, \\ EDVALDO APARECIDO AMARAL DA SILVA³ , JOSÉ MÁRCIO ROCHA FARIA4.
}

\begin{abstract}
RESUMO - O teste de raios X é uma ferramenta útil para avaliar a qualidade física de sementes florestais, que pode ser afetada pela ocorrência de sementes vazias, infestação por insetos e alterações físicas. Objetivou-se, com este estudo, verificar a eficiência do teste de raios X na avaliação dos danos internos em sementes de Eugenia pleurantha, bem como examinar a consequiência destes danos na germinação. Sementes de Eugenia pleurantha foram colocadas em suportes de isopor e expostas a diversas intensidades de radiação $(35,45,50$ e $60 \mathrm{Kvp})$, com duração de 45 e 60 segundos para determinar o padrão de raios X. De acordo com a anatomia visualizada nas radiografias, as sementes foram classificadas em Sementes Cheias e Sementes Infestadas. Em seguida, as sementes foram submetidas ao teste de germinação em substrato sobre areia a $30^{\circ} \mathrm{C}$ sob luz branca constante. A intensidade de radiação de $50 \mathrm{Kvp}$ no tempo de exposição aos raios X de 60 segundos permitiu a visualização nítida dos danos internos causados por infestação de insetos nas sementes. Os danos internos causados por larvas observados nas radiografias impedem a germinação das sementes de Eugenia pleurantha.
\end{abstract}

Termos para indexação: Danos internos, germinação, sementes florestais.

EVALUATION OF SEED QUALITY IN EUGENIA PLEURANTHA - (MYRTACEAE) BY X RAY TEST.

\begin{abstract}
The X ray test is a useful tool to evaluate seed physical quality, thatt can be affected by the occurrence of empty seeds and the presence of insects and physical alterations. The objective of this study was also to investigate the efficiency of the $\mathrm{X}$ ray test in the evaluation of internal damage in seeds of Eugenia pleurantha, as well as to examine the consequence of this damage in the germination. Seeds of Eugenia pleurantha were placed in supports and exposed to several radiation intensities $(35,45,50$ and $60 \mathrm{Kvp})$ for 45 and 60 seconds to determine the $\mathrm{X}$ ray pattern. According to the anatomy visualized in the $\mathrm{X}$ rays, the seeds were classified as full seeds and infested seeds. The seeds were submitted to the germination test with sand as substratum placed at $30^{\circ} \mathrm{C}$ under continuous light. The $50 \mathrm{Kvp}$ radiation intensity and a 60 second exposure to the $\mathrm{X}$ rays allowed clear visualization of the internal damage caused by the presence of insects in the seeds. The internal damage caused by insects prevented Eugenia pleurantha seed germination.
\end{abstract}

Index terms: internal damage, germination, forest seeds.

\footnotetext{
1 Submetido em: 09/03/2006. Aceito para publicação em. 05/07/2007. Parte da dissertação de mestrado do primeiro autor apresentada à Universidade Federal de Lavras (UFLA).

2 Eng. Agrônoma, Msc. Doutoranda em Engenharia Florestal - UFLA, CEP37200-000. Lavras, MG. tmasetto@gmail.com.

3 Eng. Agrônomos, Drs Profs. - UFLA, 37200-000. Lavras, MG.

4 Eng. Florestal, Dr. Prof. - UFLA, 372000-000. Lavras, MG.
} 


\section{INTRODUÇÃO}

$\mathrm{O}$ teste de raios $\mathrm{X}$ é um método não destrutivo usado para análise das características internas das sementes, que compreendem a anatomia e os defeitos internos, além de mudanças morfológicas que ocorrem durante a maturação e a germinação (ISTA, 1991). Esse teste vem sendo utilizado com várias finalidades, como visualização de danos mecânicos ou causados por insetos (Battisti et al., 2000) ou ainda decorrentes de outros fatores adversos pré e póscolheita, detecção de anormalidades em embriões, determinação do estádio de desenvolvimento das sementes (Machado, 2002). A técnica de raios X pode também ser aplicada na identificação de embriões mutantes (Bino et al., 1993) e na seleção de sementes cheias de aveia (Craviotto et al., 2002), aroeira (Machado e Cícero, 2003), candeia (Tonetti, 2004) e ipê (Oliveira et al., 2004).

As espécies florestais são caracterizadas pela grande ocorrência de predação, sementes vazias e má formação do embrião, entre outros. Dessa forma, o teste de raios X é recomendado pela ISTA (1999) como uma técnica promissora no controle de qualidade de sementes das espécies arbóreas.

Embora exista a necessidade de conservação de forma ex situ das espécies florestais nativas, as limitações em razão do pouco conhecimento referente às características morfológicas das sementes de tais espécies são um entrave para o processo de conservação. Dessa forma, há a necessidade de aprimorar as técnicas referentes à avaliação da qualidade de sementes das espécies florestais nativas para que o processo de armazenamento e propagação seja realizado com sucesso.

Muitas espécies do gênero Eugenia, pertencentes à família Myrtaceae, apresentam potencial nos programas de recomposição ambiental, pela capacidade de recuperação não somente da flora, como também da fauna por atraírem pássaros e outros animais (Maluf et al., 2003). As espécies pertencentes a esse gênero também são caracterizadas pelo valor comercial, nutritivo e potencial farmacológico que apresentam (Silva et al., 2003).

A Eugenia pleurantha é conhecida como pitanga-domato e cafezinho e tem ocorrência no sudeste e no sul do Brasil (Davide et al., 1995). É uma espécie propagada por sementes, portanto, a utilização de sementes com alta qualidade é essencial para o emprego desta espécie em programas florestais.

A aplicação da técnica de raios $X$ pode contribuir para o controle da qualidade das sementes de E. pleurantha, já que não existem estudos na literatura sobre a metodologia para realização do teste nesta espécie. Todavia, a eficiência do teste depende de procedimentos específicos para a espécie em estudo, como a determinação do melhor tempo e intensidade de radiação a que as sementes ficam expostas durante a execução do teste.

Diante do exposto, objetivou-se, com este trabalho, estudar a eficiência do teste de raios $\mathrm{X}$ na avaliação dos danos internos em sementes de Eugenia pleurantha, bem como verificar a conseqüência destes danos na germinação.

\section{MATERIAL E MÉTODOS}

Foram coletados frutos maduros com auxílio de podão e lona plástica de 15 matrizes na região de Lavras, localizada ao sul de Minas Gerais (21 $\left.{ }^{\circ} 14^{\prime} \mathrm{S}, 45^{\circ} 00^{\prime} \mathrm{W}\right)$. Após a coleta, os frutos permaneceram armazenados na câmara fria $\left(8^{\circ} \mathrm{C} /\right.$ 45\%UR) do Laboratório de Sementes Florestais do Departamento de Ciências Florestais da Universidade Federal de Lavras (UFLA), MG, durante dois meses. O beneficiamento consistiu da maceração dos frutos maduros em peneira, sob água corrente de modo a separar as sementes dos resíduos e secagem das sementes à sombra até que o excesso de água fosse eliminado, de acordo com as recomendações de Davide et al. (1995).

$\mathrm{O}$ grau de umidade foi determinado pelo método da estufa a $103^{\circ} \pm 2^{\circ} \mathrm{C}$ por 17 horas (Brasil, 1992), com quatro repetições de dois gramas de sementes. Os resultados foram calculados com base no peso das sementes úmidas.

Inicialmente, foi realizado o teste de germinação com quatro repetições de 25 sementes, as quais foram desinfestadas com hipoclorito de sódio $2 \%$ por 2 minutos e colocadas sobre areia autoclavada dentro de caixas plásticas, em câmara $\mathrm{BOD}$ sob $30^{\circ} \mathrm{C}$ e luz constante. Para a realização do teste de raios $X$, primeiramente foi determinada a melhor combinação de intensidade de radiação (35, 45, 50 e $60 \mathrm{Kvp}$ ) e tempo de exposição (45 e 60 segundos). Utilizou-se o equipamento Faxitron HP, modelo 43855AX.

Após a escolha das melhores intensidades e tempos de 
exposição, o teste de raios $\mathrm{X}$ foi realizado utilizando-se uma amostra aleatória de 400 sementes posicionadas sobre suporte de isopor. Em seguida, de acordo com a visualização da anatomia interna pelas radiografias, as sementes foram classificadas como sementes cheias e sementes infestadas (com danos causados por larvas ou que apresentavam larva no interior das sementes). As sementes foram então identificadas e submetidas ao teste de germinação da mesma forma citada anteriormente. O teste teve a duração de 90 dias e os números de sementes com protrusão radicular, plântulas normais e sementes mortas foram anotadas.

Os dados obtidos no teste de germinação foram submetidos à análise de variância e as médias dos tratamentos comparadas pelo Teste de Tukey, usando o programa estatístico SISVAR.

\section{RESULTADOS E DISCUSSÃO}

As sementes de Eugenia pleurantha apresentaram grau de umidade de $35,5 \%$ e germinação inicial de $87 \%$. A combinação de $50 \mathrm{Kvp}$ por 60 segundos foi considerada como a melhor combinação de intensidade de radiação e tempo de exposição das sementes à radiação, por permitir nítida visualização das estruturas internas das sementes e possibilitar a identificação dos danos que ocorrem por infestação nas sementes (Figura 1D). As demais combinações testadas não possibilitaram a observação dos danos ou larvas presentes nas sementes.

Contudo, não foi possível inferir sobre a presença de anormalidades relacionadas à morfologia do embrião como nos trabalhos realizados com sementes de milho (Cícero e Banzatto Junior, 2003), canafístula (Oliveira et al., 2003), aroeira-branca (Machado \& Cícero, 2003) e ipê-roxo e amarelo (Oliveira et al., 2004), em razão ao alto grau de umidade apresentado pelas sementes reduzir a densidade óptica dos tecidos (Simak, 1991). Essa característica implica no baixo potencial de resolução na diferenciação dos formatos e detalhes internos das sementes (Figura 1C). A habilidade de retenção de água pelas sementes viáveis em relação às sementes mortas permite distinção entre as densidades radiográficas das mesmas e, dessa forma, a avaliação de sementes não germinadas ao final do teste de germinação (Simak et al., 1989).
Porém, ao executar o teste de raios $\mathrm{X}$ com sementes submetidas à desidratação (5,0\% de umidade), estudos preliminares indicaram que não foi possível observar os detalhes da morfologia interna das sementes de $E$. pleurantha.

A dificuldade de observação dos detalhes das características morfológicas do embrião também pode ser associada ao fato do embrião de Eugenia ser conferruminado, conforme descrito por Barroso (1991), ou seja, os cotilédones apresentam-se aderidos, sem vestígio de eixo hipocótilo-radícula, sugerindo a presença de tecido meristemático não diferenciado.

De acordo com os resultados do teste de germinação (Tabela 1), constatou-se que nem todas as sementes da categoria sementes cheias (Figura 1C) $(68,75 \%)$ germinaram e formaram plântulas normais (Figura 1E). A presença de sementes mortas, geralmente, ocorre por apresentarem-se em estádios mais avançados de deterioração (Van der Burg, 1994).

Observou-se que 31,0\% das sementes correspondiam à categoria sementes infestadas. As larvas presentes nas sementes (Figuras 1B e 1D) foram identificadas, no Departamento de Entomologia da UFLA, como pertencentes à ordem Coleóptera, gênero Bruchids. A detecção das larvas não é presumível pela visualização externa das sementes, evidenciando a importância do teste de raios X para diagnosticar material infestado, assim como relatado para sementes do gênero Cassia (Kapur et al., 2002).

Os resultados de germinação referentes à categoria sementes infestadas (Tabela 1) revelaram que a presença de larvas e de danos causados pelas mesmas resultou em sementes mortas (Figura 1F). Pela análise radiográfica, também foi observado que as larvas ocuparam mais de $50 \%$ dos tecidos internos das sementes e, provavelmente, a perda da capacidade germinativa das sementes ocorreu devido ao fato das larvas terem consumido as reservas das sementes ou, mesmo, causarem danos severos ao eixo embrionário. Resultados semelhantes foram encontrados por Battisti et al. (2000) em sementes de cipreste (Cupressus sempervirens L). De acordo com estes autores, o nível de infestação das sementes afetou significativamente a germinação das sementes e está diretamente relacionado com a disseminação de fungos na semeadura desta espécie. 

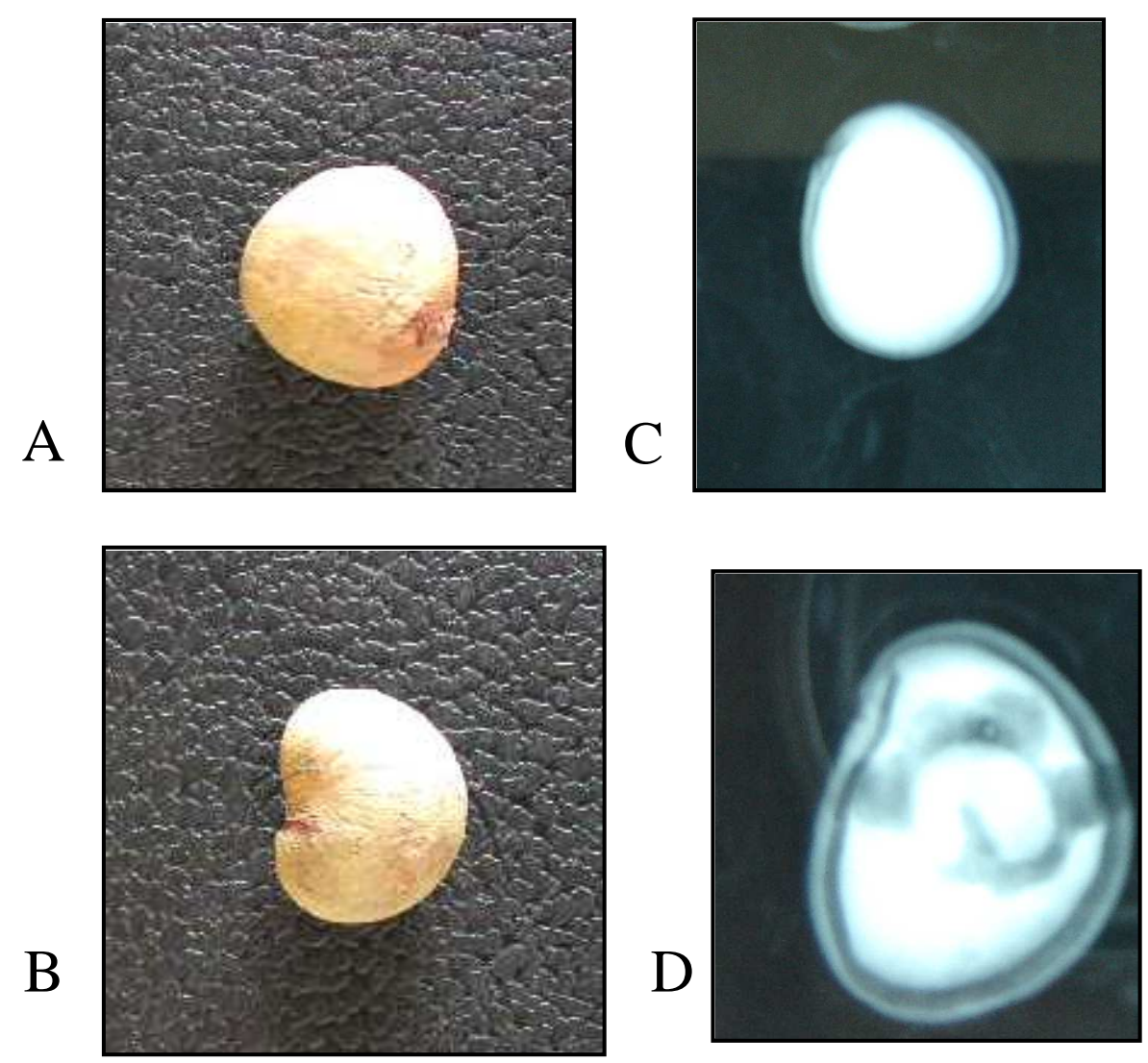
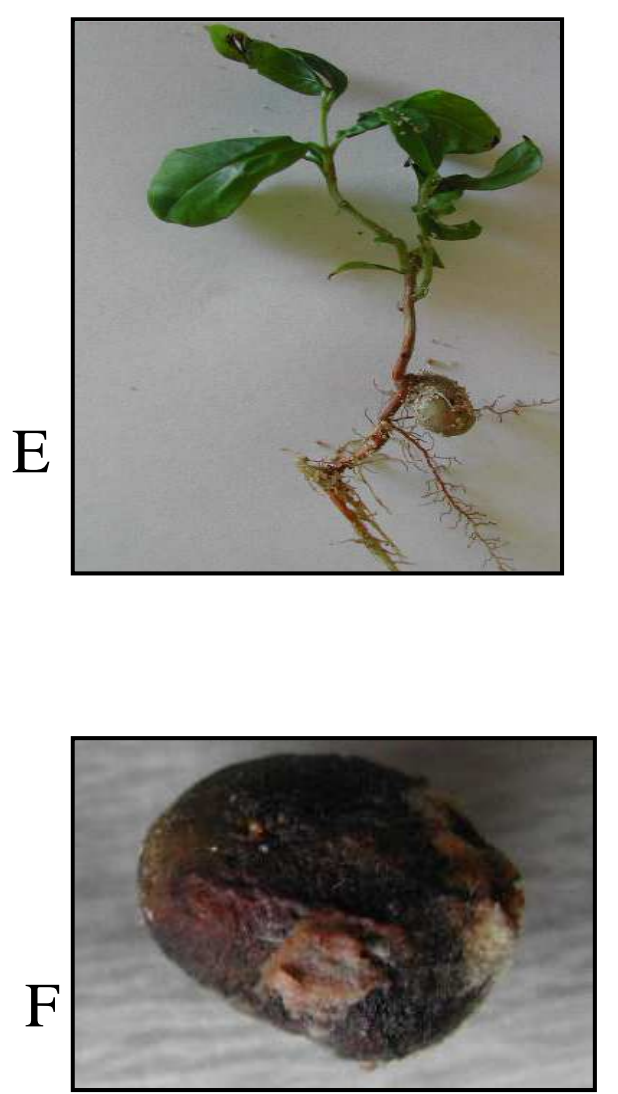

FIGURA 1. Sementes de Eugenia pleurantha visualmente intactas (A, B), classificadas pela análise radiográfica, de acordo com a anatomia interna, em Semente Cheia (C) e Semente Infestada (D). (E) - plântula normal originada de uma semente cheia; (F) - semente morta ao final do teste de germinação com sementes infestadas. UFLA, Lavras, MG, 2005.

TABELA 1. Porcentagem de germinação para cada categoria de sementes de Eugenia pleurantha, identificadas pelo teste de raios X. PR-protrusão de radícula, PN-plântulas normais, SM-sementes mortas. UFLA, Lavras, MG, 2007.

$\begin{array}{llll}\text { Categoria } & \text { PR } & \text { PN } & \text { SM }\end{array}$

$\begin{array}{cccc}\text { Cheias } & 34 \mathrm{a} & 35 \mathrm{a} & 31 \mathrm{a} \\ \text { Infestadas } & 0 \mathrm{~b} & 0 \mathrm{~b} & 100 \mathrm{~b}\end{array}$


A presença de sementes infestadas por insetos tem o mesmo efeito prejudicial sobre a qualidade do lote que a presença de sementes vazias. Sementes que contêm excrementos de insetos e larvas são fáceis e rapidamente detectadas por meio da análise radiográfica e, dessa forma, é possível prevenir a transferência de sementes contendo insetos maléficos de uma região para outra (ISTA, 1991).

Assim, o teste de raios $\mathrm{X}$ foi eficiente em estudos relacionados às sementes de Eugenia pleurantha, auxiliando na seleção de lotes e reduzindo a obtenção de sementes infestadas ou que apresentem danos causados por insetos.

\section{CONCLUSÕES}

$\mathrm{O}$ teste de raios $\mathrm{X}$ é eficiente na avaliação de danos causados por infestação em sementes de Eugenia pleurantha.

A presença de larvas e de danos causados pelas mesmas afeta a germinação, reduzindo a qualidade do lote de sementes desta espécie.

\section{REFERÊNCIAS}

BARROSO, G. M.; MORIM, M.P.; PEIXOTO, A. L.; ICHASO, C.L.F. Sistemática de angiospermas do Brasil. Viçosa: UFV, 1991. 443 p.

BATTISTI, A.; CANTINI, R.; FECI, E.; FRIGIMELICA, G.; GUIDO, M.; ROQUES, A. Detection and evaluation of seed damage of cypress, Cupressus sempervirens L., in Italy. Seed Science and Technology, Zurich, v. 28, n. 3, p.729-738, 2000.

BINO, R.J.; AARTSE, J.W.; BURG, W.J. van der. Non-destructive Xray of Arabidopsis embryo mutants. Seed Science Research, Wallingford, v.3, n.2, p.167-170, June 1993.

BRASIL. Ministério da Agricultura e Reforma Agrária. Regras para análise de sementes. Brasília: SNDA/DNDV/CLAV, 1992. 365p.

CÍCERO, S.M.; BANZATTO JUNIOR, H.L. Avaliação do relacionamento entre danos mecânicos e vigor, em sementes de milho, por meio da análise de imagens. Revista Brasileira de Sementes, Brasília, v. 25, n. 1, p. 25-28, 2003.

CRAVIOTTO, R.M.; YOLDJIAN, A.M.; SALINAS, A.R.; ARANGO, M.R.; BISARO, V.; MATURO, H. Description of pure seed fraction of oat through usual evaluations and radiographic images. Pesquisa Agropecuária Brasileira, Brasília, v. 37, n. 8, p. 1183-1188, Ago. 2002.

DAVIDE, A.C.; FARIA, J. M.R.; BOTELHO, S.A. Propagação de espécies florestais. Belo Horizonte: CEMIG/UFLA/FAEPE; Lavras: UFLA, 1995. 41p.
INTERNATIONAL RULES FOR SEED TESTING - ISTA. Seed Science and Technology, Zurich, 1999. 333p. Supplement.

INTERNATIONAL RULES FOR SEED TESTING - ISTA. Tree and shrub seed handbook. Zurich, Switzerland, 1991. paginação irregular.

KAPUR, M.L.; SHASHI-BHALLA; VERMA, B.R.; BHALLA, S. Bruchids associated with Cassia spp. seeds and their quarantine significance. Indian Journal of Entomology, New Delhi, v. 64, n. 4 , p. 471-47, 2002

MACHADO, C.F. Metodologia para a condução do teste de germinação e utilização do teste de raios $X$ para a avaliação da qualidade de sementes de aroeira-branca (Lithraea molleoides (Vell.) (Engl.). 2002. 51f. Dissertação (Mestrado)- Escola Superior de Agricultura Luiz de Queiroz, Piracicaba, SP.

MACHADO, C.F.; CICERO, S.M. Aroeira-branca (Lithraea molleoides (Vell.) Engl. - Anacardiaceae) seed quality evaluation by the X-ray test. Scientia Agricola, Piracicaba, v.60, n.2, p. 393397, abr./jun. 2003.

MALUF, A.M.; BILIA, D.A.C.; BARBEDO, C.J. Drying and storage of Eugenia involucrata DC. Seeds. Scientia Agricola, Piracicaba, v. 60, n. 3, p. 471-475, July/Sept. 2003.

OLIVEIRA, L.M.; CARVALHO, M.L.M.; DAVIDE, A.C. Utilização do teste de raios-X na avaliação da qualidade de sementes de canafístula (Peltophorum dubium (Sprengel) Taubert). Revista Brasileira de Sementes, Brasília, v. 25, n. 1, p. 116-120, 2003.

OLIVEIRA, L.M.; CARVALHO'M.L.M., GUIMARÃES, R.M., MASETTO T.E. Avaliação da qualidade de sementes de Tabebuia Serratifolia Vahl Nich. E T. Impetiginosa (Martius Ex A. P. De Candolle) Standley - (BignoniacEAE) pelo teste de raios X. Revista Brasileira de Sementes, Brasília, v. 26, n. 2, p. 138-143, dez. 2004

SILVA, C.V.; BILIA, D.A.C.; MALUF, A.M.; BARBEDO, C.J. Fracionamento e germinação de sementes de uvaia (Eugenia pyriformis Cambess. -Myrtaceae) Revista Brasileira de Botânica, São Paulo, v. 26, n. 2, p. 213-221, 2003.

SIMAK, M. Testing of forest tree and shrub seeds by $\mathrm{X}$ radiography. In: GORDON, A.G.; GOSLING, P.; WANG, B. S. P. Tree and shrub seed handbook. Zürich: ISTA, 1991.

SIMAK, M.; BERGSTEN, U.; HENRIKSSON, G. Evaluation of ungerminated seeds at the end of germination test by radiography. Seed Science and Technology, Zürich, v. 17, p. 361-369, 1989.

TONETTI, O. A.O. Melhoria da qualidade física e estudo da germinação de sementes de candeia (Eremanthus incanus (Less.) Less. e Eremanthus erythropappus (DC.) Mac. Leish). 2004. 81f. Dissertação (Mestrado em Ciências Florestais) - Universidade Federal de Lavras, Lavras, MG.

VAN DER BURG, W. J.; AARTSE, J.W.; VANZWOL, R.A.; BINO, R.J. Predicting tomato seedling morphology by X-ray analysis of seeds. Journal of the American Society for Horticultural Science, Alexandria, v.119, n. 2, p. 258-263, Mar. 1994. 